\title{
An Algorithm for Mapping ER Schema in to XML DTD with Recursion
}

\author{
Pushpa Suri, PhD \\ Computer Science Department, Retd. Professor \\ Kurukshetra University
}

\author{
Divyesh Sharma \\ Compute Science Department Research scholar \\ Kurukshetra university
}

\begin{abstract}
XML has emerged as a standard for data exchange over the internet.Relational databases provides a mature technology to store these documents. In this paper we transform ER diagram of relational databases in to DTD of XML documents. We provide an algorithm that translates ER diagram with cycles in to recursive DTDs. This algorithim translates each component of ER diagram in to components of DTDs along with cycles in ER diagram to generate recursive DTD's
\end{abstract}

\section{Keywords}

XML, DTD , Relational Databases.

\section{INTRODUCTION}

XML is a mark up language that is widely used for data exchange over the internet now a days. The data in XML is self describing. To specify the structure and syntax of XML documents DTD's are used. So DTD (Document type definition) defines the structure of an XML document. It contains a root element which is used to specify the root of an XML document. It contains elements, attributes and values. Storage of XML document in relational data bases is wide area of research for researchers now a days. We can store XML documents in relational data bases either in schema based or schema less manner. Several work [6] [7][8][9] [10] has been done in this area. Since relational data bases provides a new mature way to store and query these XML documents.In this paper we deal with the reverse area i.e. to convert relational schemes in to XML DTD's. In this paper, we describe how relational schema is converted in to DTD of XML document. We use ER diagram which is representation of relational view. IN ER diagrams, entities are related by relationships. We show how the elements of ER diagram are mapped in to the elements of DTD's. Our Algorithm also handles cycles in ER diagram that will generate recursive DTD's.

\section{RELATED WORK}

Lee [1] proposed a method of nesting based translation of relational schemes in to XML DTD's. He used nest operator for the transformation of $*$ and + operator of DTD. [2] Proposed automatic generation of DTD from ER schema. They transform every component of the ER diagram e.g. Entities, weak entities, derived attributes, relationships in to the component of DTD e. g. elements and attributes.[3] Used simple transformation for exporting the relational schema in to DTD. They transform entities in to elements and attributes into attributes and relation in to an operator element mode of ER diagram in to XML DTD. [4] supports the nested DTD generation from flat relational view. They proposed nested view of relational tables and then transform nested view into XML DTD. [5] Used the concept of primary and foreign key to transform relational schema in to DTD.

\section{MAPPING RULES}

Algorithm 1 Mapping of ER Schema in to DTD.

Input : ER Schema

Output : XMLDTD.

Step1.Map each entity of relational schema in to element.

Step2.Map each attribute of relational schema in to attribute.

Step3 Map each entity with cardinality in to element with the following operator.
(i)
(ii) $\quad 1 \ldots * \rightarrow *$
(iii) $\quad 0.1 \rightarrow$ ?

Step 4. Map each foreign key attribute in to KEYREF attribute of DTD.

Step 5.

5.1 If there are cycles in the ER diagram then transform each entity in the cycle in to an element.

5.2 Cardinality of an element in XML DTD is the highest cardinality of the entity in ER Diagram.

5.3 Map the cardinality according to the rule 3 .

Step 1 of the algorithm maps entity to the element. Step 2 maps attributes to the attributes. Step 3 maps cardinality of the entity to the operators of the elements of the DTD. Step 4 deals with KEYREF attribute of the DTD .Step 5 deals with cycles. In ER diagram. If there are cycles in the relationships between entities then it will generate recursive DTDs having elements with the operators according to the step 2 .

\section{A CASE STUDY}

We have given an example of Customer Database. Figure 1 presents ER schema of Customer Database .Figure 2 presents XMLDTD which is generated according to the algorithm described above. 


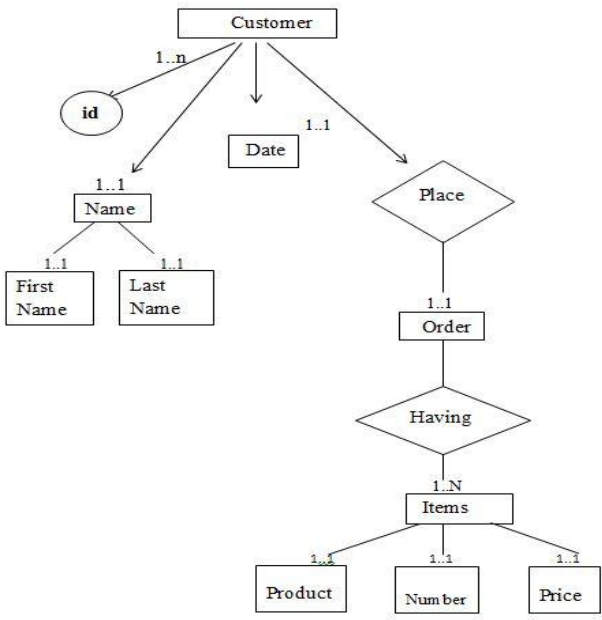

Fig 1: An Example of ER Schema of Customer Database.

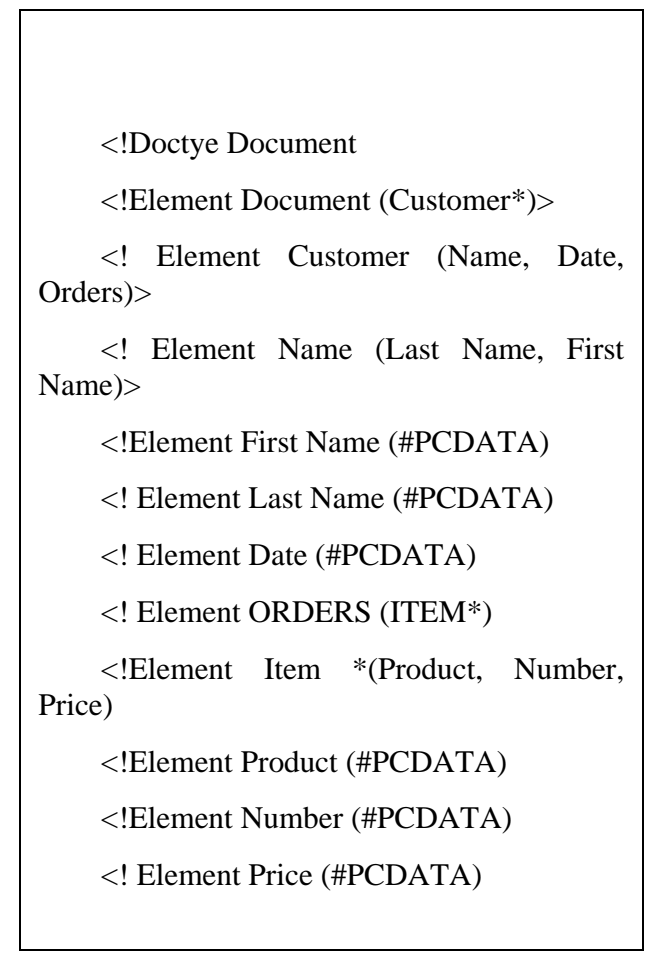

Fig 2: An Example of Generated XML DTD .

Figure 3 presents ER diagram of Employee database with cycle. In this case there are two relationships between Employee and Department Entities. Works For Relationship Exists between Employee and Department and Managed by Relationship exists between Department and Employee.

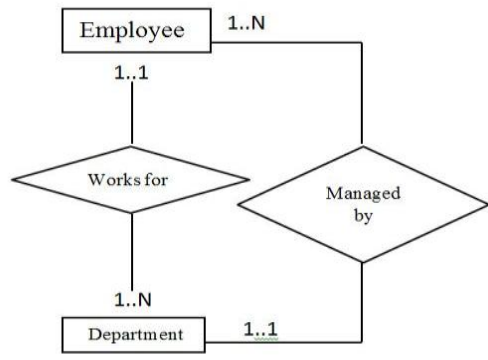

Fig 3: An Example of ER Schema with cycle.

According to Step 5 of Mapping rules the generated DTD is having recursion. It will becomes:

\section{$<$ !Element Employee (Department*)}

$<$ !Element Department (Employee*)

\section{CONCLUSION}

In this paper, cycles in ER diagram are discussed which Has not been discussed before. We presents an algorithim to generate recursive DTDs. In future work We will implement our algorihtim using java platform and compare this algorithim with NET \& COT algorithim given by LEE[1].

\section{REFERENCES}

[1] Lee, D., Mani, M.,Chiu .F ,Chu, W 2002,. Net \& CoT : Translating relational schema in to XML schema using semantic constraints. ., Proc of CIKM ,pages 282-291.

[2] Kliener C.,Lipeck U. ,2001. Automatic generation of XML DTD'S From Conceptual Database Schema."Web Databases" Work shop of the annual conference of the German and Austrian Computer Soceities in Vienna.

[3] Li Zhou, Jue Wang , 2010, A Method for exporting Relational Schema in to Document tye Dfination , IEEE.

[4] Nasir M , Ibrahim H , 2007 , Genererated Nested XML DTD From non normalized Relational Views, IJCSNS.

[5] F Laforest , M.Boumediene 2007 , Automatic Construction of XML Documents Models from a relational data models. IEEE.

[6] J.Shanmugasundaram, K. Tufte, C. Zhang, G.He, D. Dewitt,J.Naughton, 1999 ,Relational Databases for Querying XML Documents:Limitations and opportunities,VLDB ,pp : 302-314.

[7] M. Atay, A Chebotko, D. L iu, S. Lu, F. Fotoubi , 2005 ,Efficient schema based XML to relational data mapping, Information systems ,Elesevier .

[8] S.Lu,Y. Sun, M.Atay,F. Fotouhi, 2003. A New inlining algorithim for mapping XML DTDS to relational schema" In Proceedings of the First International Workshop on XML Schema and Data Management, in conjuction with the $22^{\text {nd }}$ ACM International Conference on Conceptual Modeling, Chicago, IL.

[9] M. YoshiKawa.,T.Amagasa,T.Shtimura, 2001. XREL: A path based approach to storage and retrieval of XML documents using relational databases.ACM Transactions on Internet Technology,1(1) ,pp:110-141.

[10] J. Qin, S.Zhao, S. Yang, W. Dau,2005 , XPEV : A Storage Approch for Well-Formed XML Documents, FKSD ,LNAI 3613, pp.360-369. 